\title{
Tumor necrosis factor receptor-II nt587 polymorphism in Chinese Han patients with ankylosing spondylitis
}

\author{
X. Li ${ }^{1}$, M. Wang ${ }^{1}$, R. Ma ${ }^{1}$, T. Zhang ${ }^{1}$, J. Liu ${ }^{1}$, J.W. Chen ${ }^{2}$ and W. Peng ${ }^{3}$ \\ ${ }^{1}$ Department of Clinical Laboratory, \\ The Second Xiangya Hospital of Central South University, \\ Changsha, Hunan, China \\ ${ }^{2}$ Department of Immunology \& Rheumatology, \\ The Second Xiangya Hospital of Central South University, \\ Changsha, Hunan, China \\ ${ }^{3}$ Department of Emergency, The Second People's Hospital of Hunan, \\ Changsha, Hunan, China \\ Corresponding author: M. Wang \\ E-mail: wangmin0000@aliyun.com
}

Genet. Mol. Res. 13 (3): 5190-5198 (2014)

Received June 27, 2013

Accepted October 20, 2013

Published July 7, 2014

DOI http://dx.doi.org/10.4238/2014.July.7.12

\begin{abstract}
We aimed to explore the association between the onset of ankylosing spondylitis (AS) and nt587 polymorphisms of the tumor necrosis factor receptor II (TNFRII) gene in the Han population of Hunan Province, China. Correlation analysis was performed in a case-control study involving 100 AS cases and 100 healthy controls. The nt587 single nucleotide polymorphism of the TNFRII gene was examined by polymerase chain reaction-restriction fragment length polymorphism. The relationship between AS and the frequencies of genotypes and alleles in TNFRII nt587 were analyzed using the SPSS software. There were 43 cases with the TNFRII nt587 T/T genotype, 32 cases with the TNFRII nt587 T/G genotype, and 25 cases with the TNFRII nt587 G/G genotype. In the 100 healthy controls, 56 subjects had the TNFRII nt587
\end{abstract}


T/T genotype, 34 had the TNFRII nt587 T/G genotype, and 10 had the TNFRII nt587 G/G genotype. The G allele frequency of the AS group was significantly higher $\left(\chi^{2}=8.734, \mathrm{P}=0.003\right)$ than that in the control group (41.0 vs 27.0\%). The odds ratio (OR) in AS cases with the TNFRII nt587 G/G genotype was 3.256, which was obviously higher than in those with $\mathrm{T} / \mathrm{G}(\mathrm{OR}=1.226)$ and $\mathrm{T} / \mathrm{T}(\mathrm{OR}=1.0)$ genotype. The polymorphism at position nt587 of the TNFRII gene was found to be associated with AS, and the TNFRII nt587 G allele may play an important role in AS susceptibility. The TNFRII nt587 G/G genotype may increase the risk of developing AS in the Hunan population.

Key words: Ankylosing spondylitis; Tumor necrosis factor; Tumor necrosis factor receptor; Gene polymorphism

\section{INTRODUCTION}

Ankylosing spondylitis (AS) is considered a chronic inflammatory disorder of the axial skeleton involving mainly the sacroiliac joint and lumbar spine (Khan, 1998; Brown, 2009). AS is a form of arthritis that primarily affects the spine, although other joints can also be involved. It causes inflammation of the spinal column (vertebrae) that can lead to severe chronic pain and discomfort. In the most advanced cases (but not in all cases), the inflammation can lead to new bone formation on the spine, causing the spine to fuse in a fixed, immobile position, which sometimes creates a forward-stooped posture.

Currently, gene polymorphism studies on the candidate genes of AS have been considered as promising molecular genetic methods to treat the disease (Boulos et al., 2005; Davis, 2005). The results of genome scan, linkage, and association analyses have suggested that the human leukocyte antigen (HLA) gene is the major genetic susceptibility locus of AS, among which the HLA-B27 gene shows the strongest association. However, increasing evidence has indicated that other genetic factors are also involved in AS susceptibility. Tumor necrosis factor-alpha $(\mathrm{TNF}-\alpha)$ is currently recognized as playing a central role in the pathogenesis of AS (Kanbe et al., 2008). Single nucleotide polymorphisms (SNPs) in TNF- $\alpha$ have been reported at many sites of this region, most of which involve changes between $\mathrm{G}$ and $\mathrm{A}$ at several positions including $-308,-238,-850$, and -863 (Mease, 2007; Qidwai and Khan, 2011). Furthermore, the function of TNF- $\alpha$ was associated with the cellular surface TNF receptors (TNFRs), TNFRI and TNFRII, which are widely expressed at the cell surface and in body fluids. The higher expression of TNF and TNFRs in AS patients (Haroon et al. 2010), and the therapeutic use of TNF blockers and TNFR-antibody fusion proteins in the treatment of AS (Henderson and Davis, 2006; Pan et al., 2008) have indicated that the TNFR genes are probably involved in the pathogenesis of the disease.

Polymorphisms of the TNFRII gene have previously been found in many diseases (Glossop et al., 2005; Horiuchi et al., 2007; McTiernan et al., 2012; Vasilopoulos et al., 2012). Although allele variations at nucleotide 587 (nt587) of the TNFRII gene have been associated with the incidence of AS (Tung et al., 2009), no study has yet evaluated the association between the TNFRII nt587 polymorphism and AS in the Chinese Han population. In this study, we investigated the association in the allele and genotype frequencies of the TNFRII nt587 polymorphism between Chinese Han patients with AS and controls of the same ethnic origin. 


\section{MATERIAL AND METHODS}

\section{Study subjects}

All outpatient and hospitalized patients diagnosed with AS in the Second Xiangya Hospital of Central South University (Changsha, China) from May 2008 to January 2009 were included in the study. One hundred patients, including 83 males (with an average age of $27.68 \pm 9.98$ years) and 17 females (with an average age of $27.12 \pm 9.69$ years), who met the 1984-modified New York criteria for AS (van der Linden et al., 1984) were enrolled in this study. In addition, 100 age- and sex-matched healthy controls, including 85 males (30.00 \pm 4.52 years of age) and 15 females $(32.00 \pm 5.45$ years of age), were selected from the healthy outpatients in the second Xiangya Hospital of Central South University. Each control was from a different family, and the families had no blood relationships with the cases. The exclusion criteria of this study included: those who were not of Chinese Han nationality, those with a family history of AS, those who received hormones or other immune-suppressing drugs for treatment within three months, those who were diagnosed with cardiovascular or cerebrovascular diseases, those with thrombosis or thromboembolic disease, diabetes, viral hepatitis, cirrhosis, severe hepatic and renal function insufficiency, severe malnutrition, thyroid disease, or pregnancy, those who underwent organ transplants, and patients with active tuberculosis.

Clinical and laboratory assessments were performed on the same day. Furthermore, acute-phase reactants, including erythrocyte sedimentation rates (ESR), C-reactive protein (CRP), and HLA B27, were also measured in these AS patients. The Bath Ankylosing Spondylitis Disease Activity Index (BASDAI) and the Bath Ankylosing Spondylitis Functional Index (BASFI) were determined at the same time.

The basic clinical characteristics of the AS patients and the controls are shown in Table 1.

\section{Table 1. Baseline clinical characteristic of the ankylosing spondylitis (AS) patients and the control group.}

\begin{tabular}{lcc}
\hline Characteristics & AS patients & Control \\
\hline Number of subjects & 100 & 100 \\
Male/female & $83 / 17$ & $82 / 15$ \\
Age & $(27.68 \pm 9.98) /(27.12 \pm 9.69)$ & $(30.00 \pm 4.52) /(32.00 \pm 5.45)$ \\
Disease duration (months) & $53.08 \pm 42.67$ & - \\
CRP $(\mathrm{mg} / \mathrm{dL})$ & $54.76 \pm 52.38$ & $5.32 \pm 3.46$ \\
ESR $(\mathrm{mm} / \mathrm{h})$ & $47.13 \pm 31.50$ & $4.17 \pm 3.22$ \\
Plt $\left(\mathrm{x} 10^{9} / \mathrm{L}\right)$ & $286.47 \pm 124.27$ & $203.35 \pm 53.22$ \\
HLA-B27 $(+)(\%)$ & $98 \%$ & - \\
BASDAI & $57.12 \pm 18.47$ & - \\
BASFI & $55.58 \pm 27.43$ & - \\
\hline
\end{tabular}

Values are reported as means \pm standard deviation; $\mathrm{CRP}=\mathrm{C}$-reactive protein; $\mathrm{ESR}=$ erythrocyte sedimentation rates; $\mathrm{BASDAI}=$ bath AS disease activity index; BASFI = bath AS functional index; HLA-B27 = human leukocyte antigen $\mathrm{B} 27$.

\section{DNA extraction}

Three milliliters peripheral venous blood were extracted in tubes containing edetic acid-sodium (EDTA-Na2). Genomic DNA was isolated from peripheral blood leukocytes of whole blood with a commercial DNA extraction kit (Beijing TianGen Biotech; Beijing, China) 
following manufacturer recommendations. The purity of the DNA products was detected by ultraviolet spectrophotometry.

\section{Polymerase chain reaction (PCR)}

Primers (forward primer 5'-ACTCTCCTATCCTGCCTGCT-3'; reverse primer 5'-TTCTGGAGTTGGCTGCGTGT-3') specified to the TNFRII gene were designed according to previous reports (Kammoun-Krichen et al., 2008; Machado et al., 2009). For each sample, PCR was performed in $20 \mu \mathrm{L}$ total volume containing $1 \mu \mathrm{L}$ genomic DNA as template, 10 $\mu \mathrm{L} 2 \mathrm{X}$ Taq PCR Master Mix (Beijing TianGen Biotech), and $1 \mu \mathrm{L} 10 \mu \mathrm{M}$ of each primer. The reaction conditions were as follows: pre-denaturation at $95^{\circ} \mathrm{C}$ for $5 \mathrm{~min}$ followed by 35 cycles at $95^{\circ} \mathrm{C}$ for $1 \mathrm{~min}, 60^{\circ} \mathrm{C}$ for $30 \mathrm{~s}$, and $72^{\circ} \mathrm{C}$ for $1 \mathrm{~min}$. A final extension step was carried out at $72^{\circ} \mathrm{C}$ for $5 \mathrm{~min}$. All PCR cycles were performed in the Applied Biosystem Cycler (ABI-2720). Five microliters product was loaded on $2 \%$ agarose gel, electrophoresis was carried out, and the gel was visualized under ultraviolet light after staining with ethidium bromide.

\section{Analysis of the TNFRII gene nt587 polymorphism}

The nt587 ( $>$ G) polymorphism of the TNFRII gene was analyzed by PCR-restriction fragment length polymorphism (RFLP) analysis.

The primer created a restriction site for the restriction enzyme Hin1II/N1aIII (\#ER1831, Lot: 00052826 Fermentas, MBI) (5'-CATG $\left.{ }^{\wedge}-3^{\prime}\right)$. Ten microliters amplified and purified PCR products were digested by $10 \mathrm{U} \operatorname{Hin} 1 \mathrm{II}$ at $37^{\circ} \mathrm{C}$ for $14 \mathrm{~h}$. The digestion products were identified by $6 \%$ sodium dodecyl sulfate-polyacrylamide gel electrophoresis (SDS-PAGE) analysis, and stained with silver nitrate.

\section{Statistical analysis}

The Cox proportional hazard model was applied to multivariate analysis of the association between the nt587 position polymorphisms of the TNFRII gene and AS susceptibility. All probability values were two-sided. The comparisons of genotypes and alleles between groups were investigated using the $\chi^{2}$ test. Statistical analysis was performed using the SPSS 17.0 software (SPSS Inc.; Chicago, IL, USA). P $<0.05$ was considered to be statistically different.

\section{RESULTS}

\section{Analysis of the PCR products}

The autoradiographs of nt587 of the TNFRII gene are shown in Figure 1. A band with 242-base pairs (bp) was identified.

\section{PCR-RFLP analysis and the accuracy of genotyping}

The results of the PCR-RFLP analysis for amplification fragments of TNFRII nt587 are shown in Figure 2. 


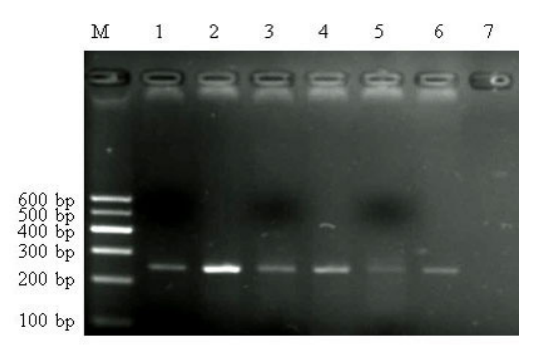

Figure 1. Agarose gel electrophoresis analysis of PCR amplification fragments for TNFR II nt587. Lane M= DNA Marker; lanes 1-6 = PCR amplification fragments of TNFR II nt587; lane 7 = blank control.

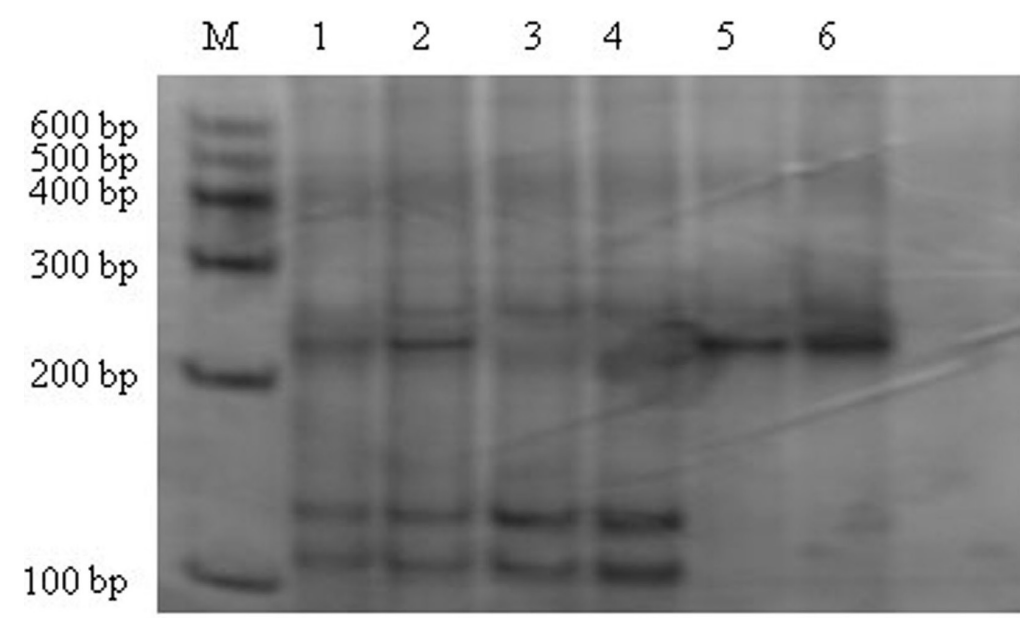

Figure 2. Electrophoresis result of PCR-RFLP for TNFR II nt587 polymorphism. Lane $M=$ DNA Marker 1; lanes $1-2=\mathrm{T} / \mathrm{G}$ genotypes; lanes 3-4 = T/T genotype; lanes $5-6=\mathrm{G} / \mathrm{G}$ genotypes.

For nt587 of the TNFRII gene, the G/G homozygous fragment could not be digested by Hin $1 \mathrm{II}$, and therefore, only one band ( $242 \mathrm{bp}$ ) was observed. For the T/T homozygous product, the presence of a Hin 1 II site was indicated by the cleavage of the amplified product into two fragments of 109 and $133 \mathrm{bp}$, respectively. The TNFRII nt587 T/G heterozygous genotype was digested into three fragments $(109,133$, and $242 \mathrm{bp})$.

The sequences of the amplified products and the partial sequence of the TNFRII nt587 are shown in Figure 3. Compared with the standard sequence (AY342040), the sequence results were consistent with the genotyping by PCR-RFLP.

\section{TNFRII nt587 polymorphism}

Table 2 shows the allele and genotype frequencies of the TNFRII gene nt587 polymorphism in AS patients and the control group. A higher frequency of the G/G homozygous fragments was observed in the AS group compared with the control group (25 vs 10\%). Using $\mathrm{T} / \mathrm{T}$ as a risk factor versus other genotypes, the estimated odds ratio (OR) for $\mathrm{G} / \mathrm{G}$ in $\mathrm{AS}$ 
patients was 3.256 [95\% confidence interval (CI): 1.417-7.497], whereas the estimated OR for $\mathrm{T} / \mathrm{G}$ in AS patients was only $1.226(95 \% \mathrm{CI}=0.656-2.221)$, and the difference was statistically significant $\left(\chi^{2}=8.196, \mathrm{P}=0.017\right)$. Furthermore, the $\mathrm{G}$ allele frequency was significantly higher in the AS group compared with controls $(41.0 \%$ vs $27.0 \%, \mathrm{P}=0.003)$. This was due to the increased frequency of homozygotes for the $\mathrm{G}$ allele in AS patients. Moreover, the $\mathrm{G}$ allele was associated with a higher risk of $\mathrm{AS}(\mathrm{OR}=1.879,95 \% \mathrm{CI}=1.214-2.861)$.

A
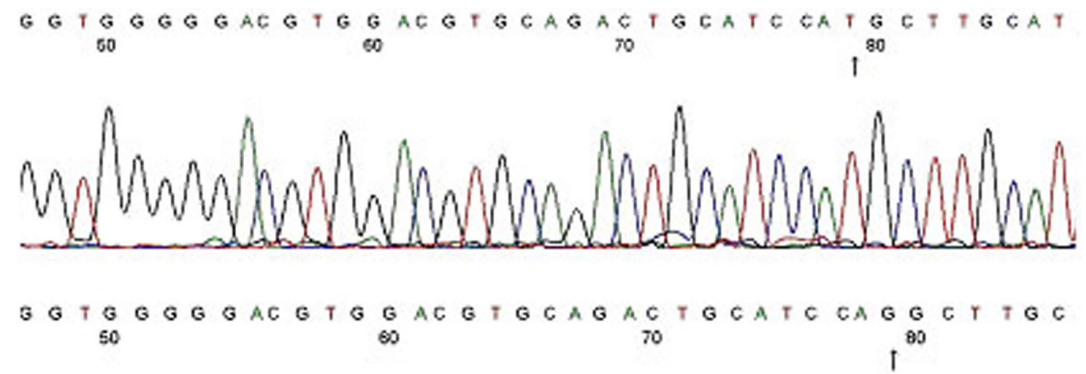

B

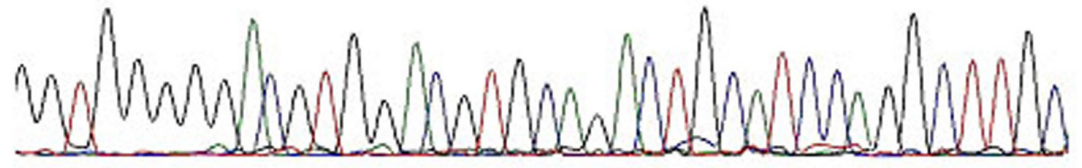

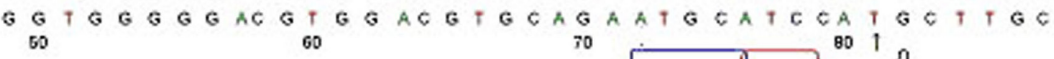

C

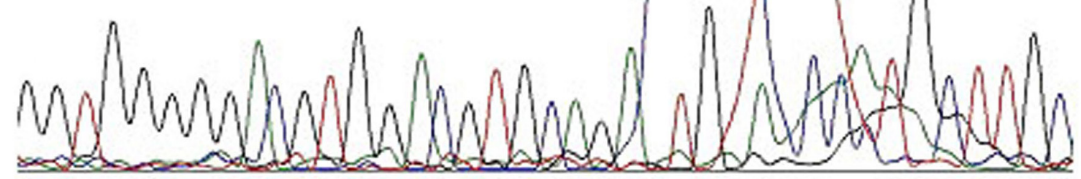

Figure 3. Sequencing map of TNFR II nt587. A. T/T genotypes; B. G/G genotypes; C. T/G genotypes.

Table 2. Distribution of alleles and genotypes in TNFR $\alpha$ nt587.

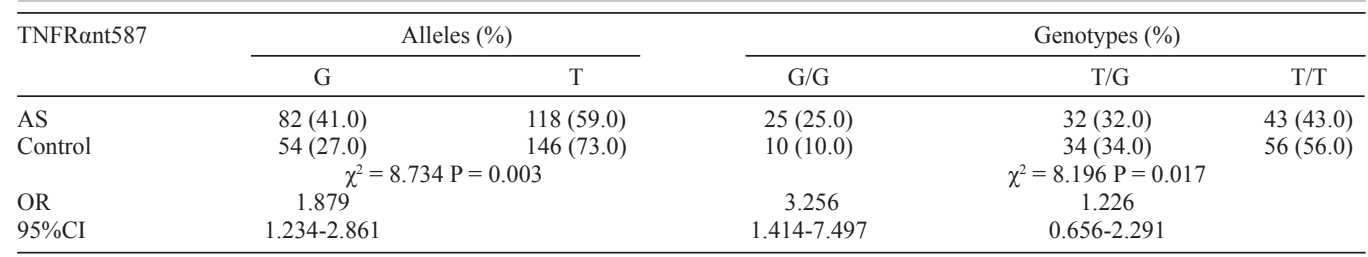

$\mathrm{AS}=$ ankylosing spondylitis.

\section{DISCUSSION}

TNF- $\alpha$, an inflammatory cytokine that plays a key role in the pathogenesis of inflammatory diseases, mediates its diverse biological effects by binding to distinct receptors 
known as TNFRI and TNFRII. TNFRII is specifically involved in the stimulation of T and B lymphocytes (Vandenabeele et al., 1995), and the exon 6 variation located at nt587 is a T to G SNP that results in the substitution of methionine by arginine at codon 196. Polymorphisms of the TNF- $\alpha$ gene have been intensively studied (Li et al., 2010; Chung et al., 2011), among which, the TNFRII nt587 polymorphism has been frequently reported in chronic autoimmune disease such as systemic lupus erythematosus (SLE), rheumatoid arthritis (RA), and alcoholic liver disease. Komata et al. (1999) demonstrated an association between SLE and the G allele of TNFRII nt587 in a Japanese population. Nevertheless, Khoa et al. (2005) reported that there was no statistical difference in the frequency of 196 TNFRII genotypes between SLE patients and healthy controls. As the results were controversial, Song et al. (2014) performed a metaanalysis to clarify the association of the TNFRII nt587 polymorphism with RA. The results showed there was no association between the TNFR2 196 R (TNFRII nt587 G) allele and RA. Furthermore, this association showed no statistical difference in European and East Asian populations. However, a significant association between the TNFR2 196 RR (TNFRII nt587 GG) genotype and RA was found in European populations. Therefore, the results indicated the association between the functional TNFR2 $196 \mathrm{M} / \mathrm{R}$ polymorphism and the susceptibility to RA might be caused by ethnicity. Machado et al. (2009) showed that no association existed between the TNFRII polymorphism and the pathogenesis of alcoholic liver disease.

To the best of our knowledge, only two association studies have been conducted between TNFR gene polymorphisms and AS to date (Tung et al., 2009; Corona-Sanchez et al., 2012). According to Corona-Sanchez et al. (2012), a higher frequency of the AA genotype was observed in the AS group in a Mexican population compared with controls ( $92 \%$ vs $79 \%)$. In addition, the A allele was increased in AS and was associated with increased genetic susceptibility for AS (OR =3.48). Another study (Tung et al., 2009) performed in a Taiwanese population found no significant differences between patients and controls in TNFRI exon I +36 (A/G) polymorphisms and in the frequencies of the $G$ allele. However, the frequency of the $G$ allele of TNFRII nt587 was significantly higher in AS patients compared to controls, suggesting that there was a significant association between AS and the nt587 polymorphism.

In our study, we focused on the nt587 polymorphism of the TNFRII gene in AS patients. The T/T genotype was identified in 43 cases, the $T / G$ genotype was identified in 32 cases, and the G/G genotype was identified in 25 cases. Accordingly, the frequencies of the $\mathrm{G}$ and $\mathrm{T}$ alleles were $41.0 \%$ and $59.0 \%$, respectively. In 2009 , to clarify the role of TNFR polymorphisms in AS in Taiwanese patients, TNFRI exon I + 36 and TNFRII exon 6 196R allele $(\mathrm{T} / \mathrm{G})$ polymorphisms were examined, which indicated that the frequencies of the 196 G/G, 196 T/G, 196 T/T genotype, $196 \mathrm{G}$ allele, and $196 \mathrm{~T}$ allele were 0, 43.6, 56.4, 21.8, and $78.2 \%$, respectively (Tung et al., 2009). In addition, in the control group, the frequencies of genotype and allele were also inconsistent with those observed in the Taiwanese population, which demonstrated that the nt587 polymorphism of the TNFRII gene varies among different regions and populations. Our results showed a significant difference in the genotype distribution in patients with $\mathrm{AS}$, in which the frequencies of the $\mathrm{G} / \mathrm{G}$ genotype and the $\mathrm{G}$ allele were higher in the AS group compared with controls $(25.0 \%$ vs $10.0 \%$ and $41 \%$ vs $27 \%$, respectively). The OR of the G/G genotype (3.256) was also much higher than that of the T/T (1.0) and $\mathrm{T} / \mathrm{G}$ (1.226) genotypes. Collectively, these results showed that the $\mathrm{G} / \mathrm{G}$ genotype of TNFRII nt587 was associated with a higher risk of AS.

Previous studies have shown that an amino acid substitution (methionine to arginine) is 
caused by the T to G SNP at TNFRII nt587, and that the amino acid substitution is responsible for its proteolytic cleavage, which produces the soluble form of TNFRII (sTNFR) (Beltinger et al., 1996; Oregon-Romero et al., 2006; Kuwahara et al., 2010). STNFR and mTNFR can compete to combine with TNF, which might affect the biological functions of TNF. Receptor shedding caused by a T to G SNP in TNFRII nt587 provided a mechanism for the downregulation of the receptor and the reduction of the protective effect of sTNFR in vivo (Aderka et al., 1992; Hooper et al., 1997). Furthermore, the mutated form was reported to diminish the ability to recruit TRAF2 (the anti-apoptotic pathway). Nevertheless, it caused no alteration of the cJun N-terminal kinase activation (the apoptotic pathway), and pre-stimulation of this mutated TNFRII gene resulted in greater activation of TNFRII-mediated apoptosis compared to the wild-type form (Till et al., 2005; Qin et al., 2012).

In conclusion, the results of this study showed a higher prevalence of the TNFRII nt587 G allele and GG genotype in patients with AS compared with matched controls. This increased prevalence might indicate an increased susceptibility to the development of AS in Chinese Han patients carrying this polymorphism. Furthermore, the substitution of $T$ to $G$ at nt587 of the TNFRII gene might contribute to a high risk of AS by affecting the content of sTNFR and the TNF signaling pathway. Further studies performed in other geographic regions are required to confirm the association between the TNFRII nt587 polymorphism and AS susceptibility in Chinese Han patients.

\section{REFERENCES}

Aderka D, Engelmann H, Maor Y, Brakebusch C, et al. (1992). Stabilization of the bioactivity of tumor necrosis factor by its soluble receptors. J. Exp. Med. 175: 323-329.

Beltinger CP, White PS, Maris JM, Sulman EP, et al. (1996). Physical mapping and genomic structure of the human TNFR2 gene. Genomics 35: 94-100.

Boulos P, Dougados M, Macleod SM and Hunsche E (2005). Pharmacological treatment of ankylosing spondylitis: a systematic review. Drugs 65: 2111-2127.

Brown MA (2009). Progress in spondylarthritis. Progress in studies of the genetics of ankylosing spondylitis. Arthritis Res. Ther. 11: 254.

Chung WT, Choe JY, Jang WC, Park SM, et al. (2011). Polymorphisms of tumor necrosis factor-alpha promoter region for susceptibility to HLA-B27-positive ankylosing spondylitis in Korean population. Rheumatol. Int. 31: 1167-1175.

Corona-Sanchez EG, Munoz-Valle JF, Gonzalez-Lopez L, Sanchez-Hernandez JD, et al. (2012). -383 A/C tumor necrosis factor receptor 1 polymorphism and ankylosing spondylitis in Mexicans: a preliminary study. Rheumatol. Int. 32: $2565-2568$.

Davis JC, Jr. (2005). Understanding the role of tumor necrosis factor inhibition in ankylosing spondylitis. Semin. Arthritis Rheum. 34: 668-677.

Glossop JR, Dawes PT, Nixon NB and Mattey DL (2005). Polymorphism in the tumour necrosis factor receptor II gene is associated with circulating levels of soluble tumour necrosis factor receptors in rheumatoid arthritis. Arthritis Res. Ther. 7: R1227-R1234.

Haroon N, Tsui FW, Chiu B, Tsui HW, et al. (2010). Serum cytokine receptors in ankylosing spondylitis: relationship to inflammatory markers and endoplasmic reticulum aminopeptidase polymorphisms. J. Rheumatol. 37: 1907-1910.

Henderson C and Davis JC (2006). Drug insight: anti-tumor-necrosis-factor therapy for ankylosing spondylitis. Nat. Clin. Pract. Rheumatol. 2: 211-218.

Hooper NM, Karran EH and Turner AJ (1997). Membrane protein secretases. Biochem. J. 321 (Pt 2): 265-279.

Horiuchi T, Kiyohara C, Tsukamoto H, Sawabe T, et al. (2007). A functional M196R polymorphism of tumour necrosis factor receptor type 2 is associated with systemic lupus erythematosus: a case-control study and a meta-analysis. Ann. Rheum. Dis. 66: 320-324.

Kammoun-Krichen M, Bougacha-Elleuch N, Makni K, Mnif M, et al. (2008). A potential role of TNFR gene polymorphisms in autoimmune thyroid diseases in the Tunisian population. Cytokine 43: 110-113. 
Kanbe K, Inoue K, Inoue Y and Suzuki Y (2008). Histological changes in bone marrow after treatment of infliximab for rheumatoid arthritis. Clin. Rheumatol. 27: 497-501.

Khan MA (1998). Ankylosing Spondylitis: The Clinical Aspects. In: The Spondylarthritides (Calin A and Taurog JD, eds.). Oxford University Press, Oxford, 27-40.

Khoa PD, Sugiyama T and Yokochi T (2005). Polymorphism of interleukin-10 promoter and tumor necrosis factor receptor II in Vietnamese patients with systemic lupus erythematosus. Clin. Rheumatol. 24: 11-13.

Komata T, Tsuchiya N, Matsushita M, Hagiwara K, et al. (1999). Association of tumor necrosis factor receptor 2 (TNFR2) polymorphism with susceptibility to systemic lupus erythematosus. Tissue Antigens 53: 527-533.

Kuwahara A, Yamamori M, Fujita M, Okuno T, et al. (2010). TNFRSF1B A1466G genotype is predictive of clinical efficacy after treatment with a definitive 5-fluorouracil/cisplatin-based chemoradiotherapy in Japanese patients with esophageal squamous cell carcinoma. J. Exp. Clin. Cancer Res. 29: 100.

Li B, Wang P and Li H (2010). The association between TNF-alpha promoter polymorphisms and ankylosing spondylitis: a meta-analysis. Clin. Rheumatol. 29: 983-990.

Machado MV, Martins A, Almeida R, Marques-Vidal P, et al. (2009). Does the simultaneous tumor necrosis factor receptor 2, tumor necrosis factor promoter gene polymorphism represent a higher risk for alcoholic liver disease? Eur. J. Gastroenterol. Hepatol. 21: 201-205.

McTiernan CF, Ramani R, Burkhead B and McNamara D (2012). The methionine 196 arginine polymorphism of the TNF receptor 2 gene (TNFRSF1B) is not associated with worse outcomes in heart failure. Cytokine 60: 838-842.

Mease PJ (2007). Adalimumab in the treatment of arthritis. Ther. Clin. Risk Manag. 3: 133-148.

Oregón-Romero E, Vázquez-Del Mercado M, Navarro-Hernández RE, Torres-Carrillo N, et al. (2006). Tumor necrosis factor receptor 2 M196R polymorphism in rheumatoid arthritis and osteoarthritis: relationship with sTNFR2 levels and clinical features. Rheumatol. Int 27: 53-59.

Pan XY, Li J, Pang J, Dai SH, et al. (2008). Clinical evaluation and radiological observation of recombinant human tumor necrosis factor receptor-Fc fusion protein in treatment of active ankylosing spondylitis. Zhonghua Yi Xue Za Zhi 88: 1408-1411.

Qidwai T and Khan F (2011). Tumour necrosis factor gene polymorphism and disease prevalence. Scand. J. Immunol. 74: $522-547$.

Qin J, Shang L, Ping AS, Li J, et al. (2012). TNF/TNFR signal transduction pathway-mediated anti-apoptosis and antiinflammatory effects of sodium ferulate on IL-1beta-induced rat osteoarthritis chondrocytes in vitro. Arthritis Res. Ther. 14: R242.

Song GG, Bae SC and Lee YH (2014). Associations between functional TNFR2 196 M/R polymorphisms and susceptibility to rheumatoid arthritis: a meta-analysis. Rheumatol. Int. [Epub ahead of print]

Till A, Rosenstiel P, Krippner-Heidenreich A, Mascheretti-Croucher S, et al. (2005). The Met-196 -> Arg variation of human tumor necrosis factor receptor 2 (TNFR2) affects TNF-alpha-induced apoptosis by impaired NF-kappaB signaling and target gene expression. J. Biol. Chem. 280: 5994-6004.

Tung CH, Lu MC, Huang KY, Yu HC, et al. (2009). Association between ankylosing spondylitis and polymorphism of tumour necrosis factor receptor II in Taiwanese patients. Scand. J. Rheumatol. 38: 395-396.

van der Linden S, Valkenburg HA and Cats A (1984). Evaluation of diagnostic criteria for ankylosing spondylitis. A proposal for modification of the New York criteria. Arthritis Rheum. 27: 361-368.

Vandenabeele P, Declercq W, Beyaert R and Fiers W (1995). Two tumour necrosis factor receptors: structure and function. Trends Cell Biol. 5: 392-399.

Vasilopoulos Y, Manolika M, Zafiriou E, Sarafidou T, et al. (2012). Pharmacogenetic analysis of TNF, TNFRSF1A, and TNFRSF1B gene polymorphisms and prediction of response to anti-TNF therapy in psoriasis patients in the Greek population. Mol. Diagn. Ther. 16: 29-34. 